\title{
Surface-assisted Laser Desorption/ionization Mass Spectrometry Analysis of the Glycolipid Biosurfactants, Mannosylerythritol Lipids, Using an Ionization-assisting Substrate
}

\author{
Tokuma Fukuoka $^{1 * \dagger}$, Sayaka Nakamura ${ }^{1 \dagger}$, Tomotake Morita ${ }^{1}$, Takayuki Ohmura ${ }^{2}$, \\ Masahiro Kotani ${ }^{2}$, Yasuhide Naito ${ }^{3}$, and Hiroaki Sato ${ }^{1}$ \\ ${ }^{1}$ Research Institute for Sustainable Chemistry, National Institute of Advanced Industrial Science and Technology (AIST), 1-1-1 Higashi, Tsukuba, \\ Ibaraki 305-8565, JAPAN \\ ${ }^{2}$ Hamamatsu Photonics K.K., 314-5 Shimokanzo, Iwata, Shizuoka 438-0193, JAPAN \\ ${ }^{3}$ The Graduate School for the Creation of New Photonics Industries, 1955-1 Kurematsu-cho, Nishi-ku, Hamamatsu, Shizuoka 431-1202, JAPAN
}

\begin{abstract}
Matrix-assisted laser desorption/ionization time-of-flight mass spectrometry (MALDI-TOF MS) is a promising tool for the screening of glycolipid-type biosurfactants (BSs) from a crude extract of microbial products. However, it is unsuitable for the detection of lower molecular weight products because the observed ions are overlapped with matrix-derived ions at lower mass range. In this study, we applied a "matrix-free" surface-assisted laser desorption/ionization mass spectrometry (SALDI-MS) analysis using a through-hole alumina membrane as an ionization-assisting substrate. Using this method, we could detect a variety of lower molecular weight products in an extract of a glycolipid BS producer with good sensitivity. In addition, the culture solution could be analyzed directly by this method.
\end{abstract}

Key words: biosurfactant, ionization-assisting substrate, MALDI-TOF MS, SALDI-MS, screening

\section{Introduction}

Biosurfactants (BSs), which are amphiphilic compounds produced by a variety of microorganisms, are more multifunctional, more environmentally and biocompatible, than chemically synthesized surfactants. Among these compounds, glycolipid-type BSs are the most promising BSs for many industrial processes because of their availability from renewable resources, such as vegetable oils and sugars, and their versatile interfacial and biochemical properties ${ }^{1,2)}$. Glycolipid-type BS molecules possess a complex bulky structure and several chiral carbons, leading to excellent surface-active properties, unique self-assembling abilities, and various biological activities. These unique chemical structures of glycolipid-type BSs are formed by biosynthesis and specific to each BS producer strains. The known structural variations of BS are limited, and the identification of novel BS producers has been greatly anticipated.

Screening for BS producers generally includes: (i)en- richment of microbes from select microbial sources, (ii) evaluation of the products in microbial cultures based on their surface-active properties, and (iii) structural determination of the BSs. Structural determination requires a large amount of product purified through time-consuming methods, such as column chromatography. Recently, we applied matrix-assisted laser desorption/ionization time-offlight mass spectrometry (MALDI-TOF/MS) to screen for glycolipid-type BS producers ${ }^{3,4)}$. MALDI-TOF MS is commonly used to identify the complex structures of organic molecules based on precise mass-to-charge ratio $(\mathrm{m} / \mathrm{z})$ measurements. This method is an excellent high-throughput screening tool that requires only a small amount of sample, and it has provided useful information on the structures of glycolipid $\mathrm{BSs}^{5-9)}$. In these studies, we subjected small amounts of crude extracts of BS producers to MALDI-TOF MS and verified this analytical tool for the rapid and reliable identification of microbial products. \footnotetext{
305-8565, JAPAN

E-mail: t-fukuoka@aist.go.jp

Accepted April 18, 2021 (received for review March 4, 2021)

Journal of Oleo Science ISSN 1345-8957 print / ISSN 1347-3352 online

http://www.jstage.jst.go.jp/browse/jos/ http://mc.manusriptcentral.com/jjocs
}

${ }^{\dagger}$ The first two authors contributor equally to this work and should be considered co-first authors.

* Correspondence to: Tokuma Fukuoka, Research Institute for Sustainable Chemistry, National Institute of Advanced Industrial Science and Technology (AIST), AIST Tsukuba Central 5-2, 1-1-1, Higashi, Tsukuba, Ibaraki

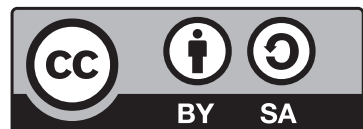


However, MALDI-TOF MS analysis is not appropriate for the detection of every BS. It is unsuitable for the detection of lower molecular weight products $(m / z<500)$ due to interference with matrix-derived ions. Additionally, tested BSs are prepared by extraction using an organic solvent, such as ethyl acetate or hexane, to remove impurities from the microbial cultures. Therefore, MALDI-TOF MS might miss lower molecular weight and more hydrophilic microbial products. Thus, in this study, we used an ionization-assisting substrate and performed surface-assisted laser desorption/ionization mass spectrometry (SALDI-MS) analysis $^{10,11}$ of culture extracts of BS producers as a matrix-free method using a through-hole alumina membrane as an ionization-assisting substrate ${ }^{12,13)}$. Mannosylerythritol lipids (MELs; Fig. 1) were selected as a target of the investigation. These are glycolipid-type BSs produced by fungal strains of the genera Pseudozyma or Ustilago ${ }^{14,15)}$. We obtained not only diacylated MELs as a main component, but also monoacylated MELs when the MEL production was carried out using glucose as the sole carbon source ${ }^{16)}$. These monoacylated MELs are more hydrophilic and of lower molecular weight than diacylated MELs and are assumed to be unsuitable for extraction from microbial cultures with ethyl acetate followed by MALDI-TOF MS analysis. Therefore, if monoacylated MELs could be detected with good sensitivity in SALDI-MS analysis of Pseudozyma yeast cultures, this method would be demonstrated to be a powerful analytical tool for the screening of producers of glycolipid BSs with a variety of chemical structures.

\section{Experimental Procedures}

\subsection{Materials and microorganisms}

All reagents and solvents were commercially available and were used as received. Pseudozyma antarctica JCM10317 was used as a MEL producer. The ionization-assisting substrate (DIUTHAME ${ }^{\circledR}$ chips) is a commercial

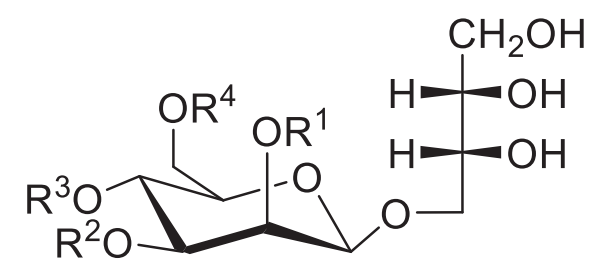

Conventional (diacylated) MEL: $R^{1}, R^{2}=$ fatty acyl chain monoacylated MEL: $\mathrm{R}^{1}, \mathrm{R}^{2}=$ either fatty acyl chain or $\mathrm{H}$ MEL-A: $R^{3}=R^{4}=A c$

MEL-B: $R^{3}=H, R^{4}=A C$

MEL-C: $R^{3}=A c, R^{4}=H$

MEL-D: $R^{3}=\mathrm{R}^{4}=\mathrm{H}$

ME: $\mathrm{R}^{1} \sim \mathrm{R}^{4}=\mathrm{H}$

Fig. 1 Chemical structures of mannosylerythritol lipids. product available from Hamamatsu Photonics K. K. (Hamamatsu, Japan). The production procedures, characteristics, and shape of the through-holes are described elsewhere ${ }^{17)}$.

\subsection{Preparation of MELs}

Mannosylerythritol lipids were prepared using glucose as the sole carbon source by the yeast strain $P$. antarctica JCM10317 as previously reported ${ }^{16)}$. Seed cultures were prepared by inoculating cells grown on the slants into growth medium ( $4 \% \mathrm{w} / \mathrm{w}$ glucose, $0.3 \% \mathrm{w} / \mathrm{w} \mathrm{NaNO}_{3}, 0.03 \%$ w/w $\mathrm{MgSO}_{4} 7 \mathrm{H}_{2} \mathrm{O}, 0.03 \%$ w/w $\mathrm{KH}_{2} \mathrm{PO}_{4}$, and $0.1 \%$ w/w yeast extract ( $\mathrm{pH} 6.0)$ ) at $25^{\circ} \mathrm{C}$ on a reciprocal shaker $(150 \mathrm{rpm}$ ) for 2 days. The seed cultures $(1 \mathrm{~mL})$ were transferred to 300-mL Erlenmeyer flasks containing $30 \mathrm{~mL}$ of basal medium containing $10 \% \mathrm{w} / \mathrm{w}$ glucose, and then incubated on a rotary shaker $(250 \mathrm{rpm})$ at $25{ }^{\circ} \mathrm{C}$ for 7 days. After 7 days of cultivation, the culture broth was extracted with an equal volume of ethyl acetate, followed by mass spectrometry analysis.

2.3 SALDI-MS analysis of extracts from culture broth containing MELs using an ionization-assisting substrate

SALDI-MS analysis was performed according to previous reports ${ }^{12,13)}$. The preparation procedures were almost identical for crude ethyl acetate extracts containing MELs from culture broth and supernatant of the culture broth of $P$. antarctica JCM10317. Sample was applied to the back side of the DIUTHAME ${ }^{\circledast}$ chip and dried. For culture broth, $c a .3$ $\mu \mathrm{L}$ of methanol was additionally applied onto the chip. Next, the chip was inverted and sodium trifluoroacetate (NaTFA) solution ( $c a .1 \mu \mathrm{L}$ ) added onto the front side of the chip and allowed to dry (Fig. 2). Mass spectra were recorded using a JMS-S3000 SpiralTOF instrument(JEOL Ltd., Akishima, Japan).

\section{Results and Discussion}

3.1 SALDI-MS analysis of MELs using an ionizationassisting substrate

In the MALDI mass spectrum of MELs (Fig. S1 in the Supporting Information), matrix and plasticizer-derived ion peaks were mostly detected in the low mass range $(\mathrm{m} / \mathrm{z}$ $<500$ ). Therefore, MALDI-TOF MS is not usually applied to the mass analysis of low molecular weight samples to avoid the interference of such peaks. In this study, to observe low mass area clearly, we carried out SALDI-MS using an ionization-assisting substrate without matrix. Crude ethyl acetate extracts of culture broths containing MELs were directly applied to the ionization-assisting substrate, and the dried sample substrate was analyzed. The obtained mass spectrum is shown in Fig. 3a. We previously reported that $P$. antarctica produces monoacylated MELs 


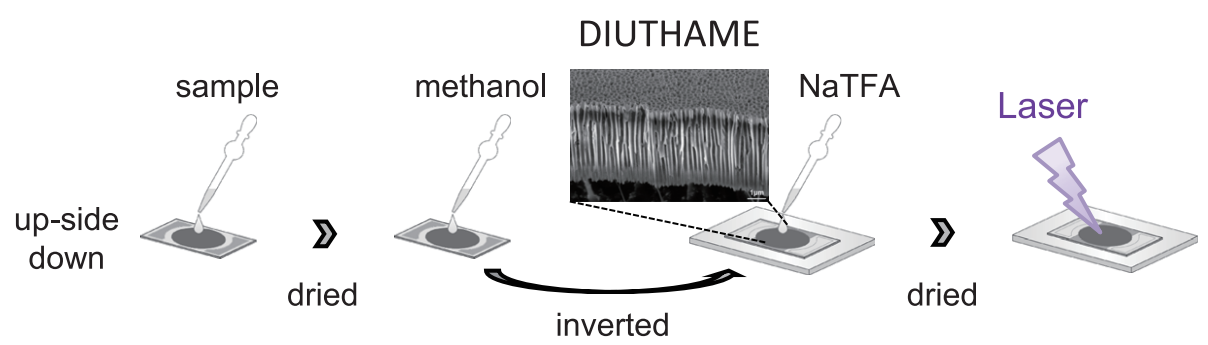

Fig. 2 Workflow for surface-assisted laser desorption/ionization mass spectrometry analysis of culture broth using a through-hole alumina membrane as an ionization substrate.

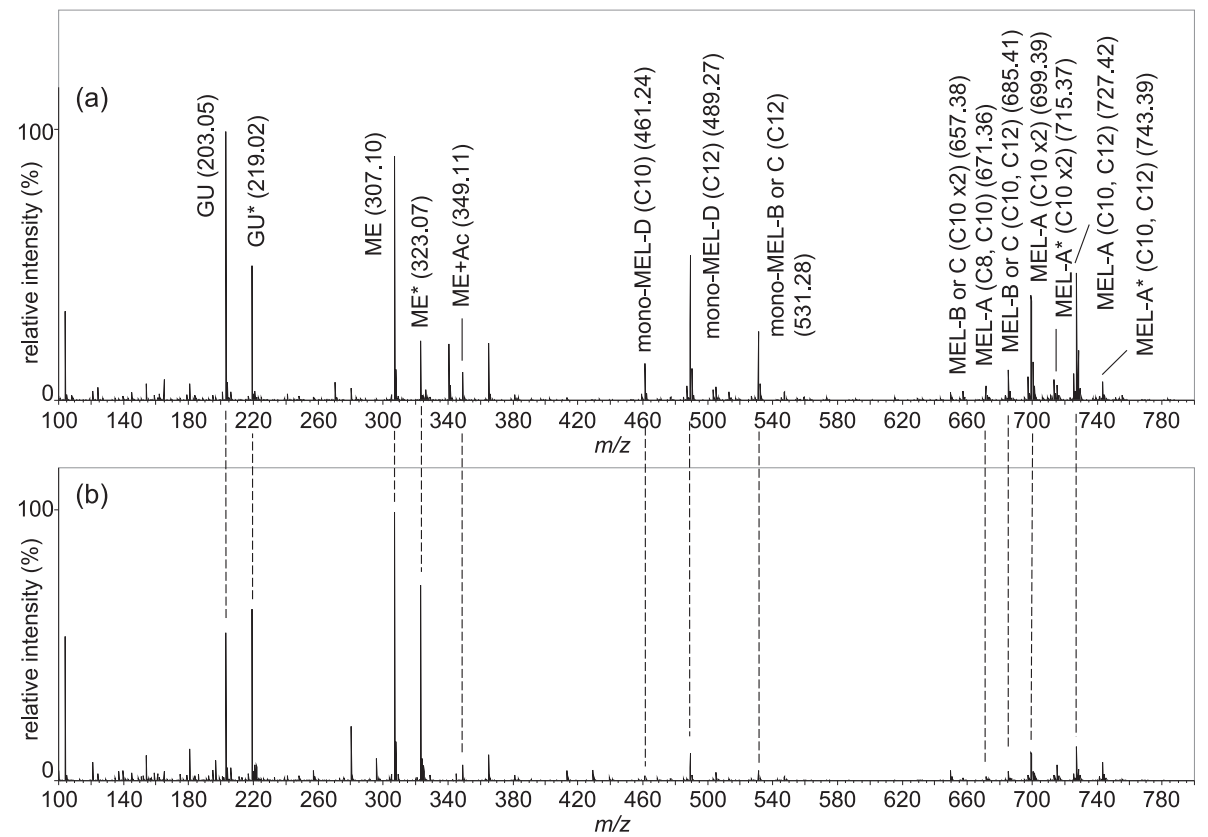

Fig. 3 Mass spectra of (a) crude ethyl acetate extracts of culture broth and (b) culture broth supernatant from Pseudozyma antarctica. Unmarked: the sodium adduct, *: the potassium adduct.

as well as conventional diacylated MELs when using glucose as the sole carbon source ${ }^{16)}$. In the mass spectrum, peaks corresponding to monoacylated and diacylated MELs were clearly detected over the ranges of $\mathrm{m} / \mathrm{z} 450-600$ and $m / z 650-750$, respectively. In addition, some peaks in the lower mass range $(\mathrm{m} / z 100-500)$ were detected. The peak at $m / z 203$ corresponded to glucose, which was present in the culture broth. The peaks at $\mathrm{m} / z 307$ and $\mathrm{m} / z 349$ were assigned to mannosylerythritol(ME) without esterified acyl groups and acetylated $\mathrm{ME}$, respectively, which were produced as intermediates in MEL biosynthesis. Peaks were observed for both the sodium and potassium adducts. These peaks have been difficult to detect by conventional MALDI-TOF MS analysis because of overlapping matrix cluster ion peaks. Because the present measurement was performed using a matrix-free method, these ion peaks could be detected without interference from matrix cluster ions.

\subsection{Direct SALDI-MS analysis of microbial cultures con- taining MELs using an ionization-assisting substrate}

DIUTHAME substrate is made of a through hole porous alumina membrane ${ }^{17)}$. When a sample solution is applied to the substrate, we expected that organic components in the solution are adsorbed and concentrated on the substrate and a solvent passes through the substrate. Therefore, we tried to perform direct mass analysis of the crude culture broth using the DIUTHAME chip without the extraction process. The culture broth supernatant was freed of yeast cells by gentle centrifugation and then applied to the ionization-assisting substrate and allowed to dry. The obtained mass spectrum is shown in Fig. 3b. The spectral pattern was almost identical to that of ethyl acetate extract of the culture broth, with differences only in the peak intensities. Thus, this method permits direct SALDI-MS analysis of the microbial culture broth without pretreatment. In addition, more hydrophilic products with lower molecular weights (e.g., ME and acetylated ME) were detected with good sensitivity from the culture broth. There was almost no differ- 


\section{T. Fukuoka, S. Nakamura, T. Morita et al.}

ence in the peak intensities between monoacylated MELs and diacylated MELs. This method is particularly suitable for the detection of microbial products that are difficult to extract from culture broth with an organic solvent. Our findings indicate that SALDI-MS is a promising highthroughput screening tool for BS producers, with which microbial products in the culture broth can be directly analyzed without pretreatment.

\section{Conclusion}

In this study, SALDI-MS analysis of glycolipid BSs, i.e., MELs, was performed for the first time using an ionizationassisting substrate without matrix. More hydrophilic and lower molecular weight intermediates of the conventional diacylated MEL biosynthesis, such as monoacylated MELs and mannosylerythritol and its derivatives, were detected without interference by matrix cluster ions. In addition, the culture broth supernatant containing MELs could be directly analyzed without pretreatment, and all extracellular products were detected. The present matrix-free method of SALDI-MS is a significant advance for screening glycolipid BS producers because all microbial products in the culture broth can be rapidly and directly detected without pretreatment, and more hydrophilic microbial products with lower molecular weight can be detected with good sensitivity.

\section{Acknowledgment}

We thank Mr. Yoshiyuki Hayashibara for his technical assistance.

\section{Supporting Information}

This material is available free of charge via the Internet at doi: 10.5650/jos.ess21084

\section{References}

1) Kitamoto, D.; Morita, T.; Fukuoka, T.; Konishi, M.; Imura, T. Self-assembling properties of glycolipid biosurfactants and their potential applications. Curr. Opin. Colloid Interface Sci. 14, 315-328(2009).

2) Mnif Inès, M.; Dhouha, G. Glycolipid biosurfactants: Potential related biomedical and biotechnological applications. Carbohydr. Res. 416, 59-69(2015).

3) Sato, S.; Fukuoka, T.; Saika, A.; Koshiyama, T.; Morita, T. A new screening approach for glycolipid-type biosurfactant producers using MALDI-TOF/MS. J. Oleo
Sci. 68, 1287-1294(2019).

4) Saika, A.; Fukuoka, T.; Mikome, S.; Kondo, Y.; Habe, H.; Morita, T. Screening and isolation of the liamocin-producing yeast Aureobasidium melanogenum using xylose as the sole carbon source. J. Biosci. Bioeng. 129, 428-434 (2020).

5) Rooney, A.P.; Price, N.P.J.; Ray, K.J.; Kuo, T.-M. Isolation and characterization of rhamnolipid-producing bacterial strains from a biodiesel facility. FEMS Microbiol. Lett. 295, 82-87(2009).

6) Kurtzman, C.P.; Price, N.P.J.; Ray, K.J.; Kuo, T.-M. Production of sophorolipid biosurfactants by multiple species of the Starmerella (Candida) bombicola yeast clade. FEMS Microbiol. Lett. 311, 140-146(2010).

7) Price, N.P.J.; Ray, K.J.; Vermillion, K.E.; Dunlap, C.A.; Kurtzman, C.P. Structural characterization of novel sophorolipid biosurfactants from a newly identified species of Candida yeast. Carbohydr. Res. 348, 33-41 (2012).

8) Wu, J.; Zhang, J.; Wang, P.; Zhu, L.; Gao, M.; Zheng, Z.; Zhan, X. Production of rhamnolipids by semi-solidstate fermentation with Pseudomonas aeruginosa RG18 for heavy metal desorption. Bioprocess Biosyst. Eng. 40, 1611-1619(2017).

9) Beck, A.; Haitz, F.; Grunwald, S.; Preuss, L.; Rupp, S.; Zibek, S. Influence of microorganism and plant oils on the structure of mannosylerythritol lipid (MEL) biosurfactants revealed by a novel thin layer chromatography mass spectrometry method. J. Ind. Microbiol. Biotechnol. 46, 1191-1204(2019).

10) Law, K.P.; Larkin, J.R. Recent advances in SALDI-MS techniques and their chemical and bioanalytical applications. Anal. Bioanal. Chem. 399, 2597-2622 (2011).

11) Guinan, T.; Kirkbride, P.; Pigou, P.E.; Ronci, M.; Kobus, H.; Voelcker, N.H. Surface-assisted laser desorption ionization mass spectrometry techniques for application in forensics. Mass Spectrom. Rev. 34, 627-640 (2015).

12) Sato, H.; Nakamura, S.; Fouquet, T.; Ohmura, T.; Kotani, M.; Naito, Y. Molecular characterization of polyethylene oxide based oligomers by surface-assisted laser desorption/ionization mass spectrometry using a through-hole alumina membrane as active substrate. Rapid Commun. Mass Spectrom. 34, e8597 (2020).

13) Sato, H.; Nakamura, S.; Fouquet, T.N.J.; Ohmura, T.; Kotani, M.; Naito, Y. Simple pretreatment for the analysis of additives and polymers by surface-assisted laser desorption/ionization mass spectrometry using a through-hole alumina membrane as a functional substrate. J. Am. Soc. Mass Spectrom. 31, 298-307 (2020).

14) Morita, T.; Fukuoka, T.; Imura, T.; Kitamoto, D. Mannosylerythritol lipids: Production and applications $J$. 
Oleo Sci. 64, 133-141(2015).

15) Yu, M.; Liu, Z.; Zeng, G.; Zhong, H.; Liu, Y.; Jiang, Y.; Li, M.; He, X.; He, Y. Characteristics of mannosylerythritol lipids and their environmental potential. Carbohydr. Res. 407, 63-72 (2015).

16） Fukuoka, T.; Morita, T.; Konishi, M.; Imura, T.; Sakai, H.; Kitamoto, D. Structural characterization and surfaceactive properties of a new glycolipid biosurfactant, mono-acylated mannosylerythritol lipid, produced from glucose by Pseudozyma antarctica. Appl. Microbiol. Biotechnol. 76, 801-810 (2007).

17) Naito, Y.; Kotani, M.; Ohmura, T. A novel laser desorp- tion/ionization method using through hole porous alumina membranes. Rapid Commun. Mass Spectrom. 32, 1851-1858(2018).

CC BY-SA 4.0 (Attribution-ShareAlike 4.0 International). This license allows users to share and adapt an article, even commercially, as long as appropriate credit is given and the distribution of derivative works is under the same license as the original. That is, this license lets others copy, distribute, modify and reproduce the Article, provided the original source and Authors are credited under the same license as the original.

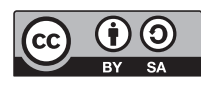

